Article

\title{
Hybrid Theory of Scattering and Its Applications
}

\author{
Anand K. Bhatia
}

Heliophysics Science Division, NASA/Goddard Space Flight Center, Greenbelt, MD 20772, USA;

Anand.K.Bhatia@nasa.gov

Received: 12 April 2018; Accepted: 7 May 2018; Published: 14 May 2018

\begin{abstract}
A number of formulations have been used to investigate scattering of low-energy electrons and positrons from various targets. The hybrid theory of scattering, which takes into account the short-range as well as the long-range correlations, and is variationally correct, is described in this article. This approach has been applied to calculate phase shifts for scattering of electrons and positrons, resonances in two-electron systems, photodetachment, and photoionization of two-electron systems. This approach has also been applied to calculate excitation of $2 s$ state of atomic hydrogen by electron impact. In photoabsorption the target can be left in $2 p$ state instead of $1 s$ state, resulting in the emission of Lyman-alpha radiation. Cross sections for this process are also calculated.
\end{abstract}

Keywords: hybrid theory of scattering

\section{Introduction and Calculations}

Scattering by single-electron systems is always of interest because the wave function of the target is known exactly. Also, scattering from atoms helps us to understand their static and dynamic properties. It also has applications in astrophysics and in the investigation of controlled thermonuclear devices. Different approaches have been applied to study scattering of electrons and positrons from various targets, to infer the feasibility of various calculations and the accuracy of their results. In other words, this is an ideal problem for testing various theories. A simple method is to assume that the wave function of the incident electron on hydrogen atom is given by $\Psi\left(\vec{r}_{1}, \vec{r}_{2}\right)=u\left(\vec{r}_{1}\right) \Phi_{0}\left(\vec{r}_{2}\right)$, where $\Phi_{0}\left(\vec{r}_{2}\right)$ is the ground state of the hydrogen atom and $u\left(\vec{r}_{1}\right)$ is the function whose asymptotic limit determines phase shift of the scattering. Morse and Allis [1] introduced exchange between electrons, writing the wave function in the form:

$$
\Psi\left(\vec{r}_{1}, \vec{r}_{2}\right)=u\left(\vec{r}_{1}\right) \Phi_{0}\left(\vec{r}_{2}\right) \pm u\left(\vec{r}_{2}\right) \Phi_{0}\left(\vec{r}_{1}\right) .
$$

The ground state function is given by:

$$
\Phi_{0}\left(\vec{r}_{2}\right)=\sqrt{\frac{Z^{3}}{\pi}} e^{-Z r_{2}}
$$

The upper sign in Equation (1) corresponds to the singlet state and the lower sign corresponds to triplet state of the system. The equation for the scattering function $u(\mathrm{r})$ (letting $r_{1}=\mathrm{r}$ ) is obtained by the Kohn variation of the functional:

$$
\left\langle Y_{L 0}\left(\Omega_{1}\right) \Phi_{0}\left(\vec{r}_{2}\right)|H-E| \Psi\left(\vec{r}_{1}, \vec{r}_{2}\right)\right\rangle=0,
$$

where $E=k^{2}-Z^{2}$ and $k^{2}$ is the kinetic energy of the incident electron, and $Z$ is the charge of the nucleus. Considering the proton stationary during the interaction, the Hamiltonian $H$ of the system in Rydberg units is given by: 


$$
H=-\nabla_{1}^{2}-\nabla_{2}^{2}-\frac{2 Z}{r_{1}}-\frac{2 Z}{r_{2}}+\frac{2}{r_{12}}
$$

In the above equation, $r_{12}=\left|\vec{r}_{1}-\vec{r}_{2}\right|$. Using Equation (3), we get the integro-differential equation:

$$
\begin{gathered}
\left.\left[\frac{d^{2}}{d r^{2}}+\frac{2(Z-1)}{r}-\frac{L(L+1)}{r}+e^{-2 Z r} 2\left(Z+\frac{1}{r}\right)+k^{2}\right)\right] u(r) \pm 4 Z^{3} e^{-Z r}\left[\left(k^{2}+Z^{2}\right) r \delta_{L 0} I_{1}\right. \\
\left.-\frac{2}{2 L+1}\left(\frac{I_{2}}{r^{L}}+r^{L+1} I_{3}\right)\right]=0 .
\end{gathered}
$$

The exchange terms with $I_{1}, I_{2}$, and $I_{3}$ are the nonlocal potentials and are functions of $\mathrm{u}(\mathrm{r})$ :

$$
\begin{gathered}
I_{1}=\int_{0}^{\infty} e^{-Z x} x u(x) d x, \\
I_{2}=\int_{0}^{r} e^{-Z x} x^{L+1} u(x) d x,
\end{gathered}
$$

and

$$
I_{3}=\int_{r}^{\infty} e^{-Z x} \frac{u(x)}{x^{L}} d x .
$$

The singlet and triplet $S$-wave phase shifts in the exchange approximation are given in Table 1. Among the different methods used is the variational principle of Kohn [2]. Schwartz [3] used this principle to calculate phase shifts of electrons and positrons scattering from hydrogen atoms. There are no bounds on phase shifts in this principle except at incident energy $k=0$. There are also singularities in this calculation. In spite of singularities, the results obtained in [3] are fairly accurate and have stood the test of time. Another extensively used method is the close-coupling approximation [4], in which the total wave function is expanded in eigenstates of the hydrogen atom. Accurate results have been obtained using the $R$-matrix formulation [5] in which most of the complications of interactions are taken into account within the inner region of a certain radius and outside this radius plane waves or Coulomb waves are used for continuum functions.

Table 1. Phase shifts (radians) for the ${ }^{1} \mathrm{~S}$ and ${ }^{3} \mathrm{~S}$ states of e-H.

\begin{tabular}{ccccccc}
\hline \multirow{3}{*}{$\boldsymbol{K}$} & \multicolumn{3}{c}{ Singlet } & \multicolumn{3}{c}{ Triplet } \\
\cline { 2 - 7 } & EA $^{\mathbf{a}}$ & PO [6] & QHQ [7] & EA $^{\mathbf{a}}$ & PO [6] & QHQ [7] \\
\hline 0.0 & 8.100 & 5.9 & & 2.349 & 1.9 & \\
0.1 & 2.396 & 2.583 & 2.55358 & 2.908 & 2.945 & 2.93853 \\
0.2 & 1.870 & 2.144 & 2.06678 & 2.679 & 2.732 & 2.71741 \\
0.3 & 1.508 & 1.750 & 1.69816 & 2.461 & 2.519 & 2.49975 \\
0.4 & 1.239 & 1.469 & 1.41540 & 2.257 & 2.320 & 2.29408 \\
0.5 & 1.031 & 1.251 & 1.20094 & 2.070 & 2.133 & 2.10454 \\
0.6 & 0.869 & & 1.04083 & 1.901 & & 1.93272 \\
0.7 & 0.744 & 0.947 & 0.93111 & 1.749 & 1.815 & 1.77950 \\
0.8 & 0.651 & 0.854 & 0.88718 & 1.614 & 1.682 & 1.64379 \\
\hline
\end{tabular}

$\mathrm{EA}^{\mathrm{a}}$ are exchange approximation phase shifts, and $k=0$ results are scattering lengths.

It has been emphasized by Wigner [8] that long range forces determine the nature of the scattering parameters at threshold. A method in which the longest-range potential proportional to $-1 / \mathrm{r}^{4}$ is taken into account is the method of polarized orbital [6,9]. This method includes the essential physics of the problem and has been widely used not only to calculate scattering parameters for electrons and positrons but also to calculate photoabsorption cross sections. Using the first-order perturbation theory 
and the dipole part of the perturbed wave function, Temkin [9] showed that the effective target wave function in the presence of the incident electron can be written as

$$
\Phi^{p o l}\left(\vec{r}_{1}, \vec{r}_{2}\right)=\Phi_{0}\left(\vec{r}_{2}\right)-\frac{\varepsilon\left(r_{1}, r_{2}\right)}{r_{1}^{2}} \frac{u_{1 s \rightarrow p}\left(r_{2}\right)}{r_{2}} \frac{\cos \left(\theta_{12}\right)}{\sqrt{Z \pi}}
$$

where

$$
\begin{gathered}
u_{1 s \rightarrow p}\left(r_{2}\right)=e^{-Z r_{2}}\left(0.5 Z r_{2}^{3}+r_{2}^{2}\right) . \\
\varepsilon\left(r_{1}, r_{2}\right)=1, r_{1}>r_{2} \\
=0, r_{1}<r_{2} .
\end{gathered}
$$

Now, the function in Equation (1) is written as:

$$
\Psi\left(\vec{r}_{1}, \vec{r}_{2}\right)=u\left(\vec{r}_{1}\right) \Phi^{p o l}\left(\vec{r}_{1}, \vec{r}_{2}\right) \pm(1 \leftrightarrow 2) .
$$

The equation for the scattering function $u_{L}\left(r_{1}\right)$ can be derived from:

$$
\left\langle Y_{L 0}\left(\Omega_{1}\right) \Phi_{0}\left(r_{2}\right)|H-E| \Psi\left(\vec{r}_{1}, \vec{r}_{2}\right)\right\rangle=0
$$

The equation for the scattering function $u_{L}(r)$ for all momenta has been given by Sloan [10]:

$$
\begin{aligned}
& \left.\left[\frac{d^{2}}{d r^{2}}+\frac{2(Z-1)}{r}-\frac{L(L+1)}{r}+e^{-2 Z r} 2\left(Z+\frac{1}{r}\right)+k^{2}\right)\right] u_{L}(r) \pm 4 Z^{3} e^{-Z r}\left[\left(k^{2}+Z^{2}\right) r \delta_{L 0} I_{1}\right. \\
& \left.-\frac{2}{2 L+1}\left\{r^{-L} I_{2}+r^{L+1} I_{3}\right\}\right]=-\frac{\alpha(Z r)}{(Z r)^{4}} u_{L}(r) \\
& \mp 4 Z e^{-Z r}\left[\frac{\delta_{L 1}}{3}\left\{-\frac{Z\left(Z^{2}+k^{2}\right)}{2} r^{3}+\left(Z^{2}-k^{2}\right) r^{2}\right\} \int_{r}^{\infty} e^{-Z x} x^{-L} u_{L}(x) d x\right. \\
& +2\left(Z r^{3}+r^{2}\right)\left\{\frac{L}{(2 L-1)(2 L+1)} r^{L-1} \int_{r}^{\infty} e^{-Z x} x^{-L-1} u_{L}(x) d x\right. \\
& \left.\left.+\frac{L+1}{(2 L+1)(2 L+3)} r^{L+1} \int_{r}^{\infty} e^{-Z x} x^{-L-3} u_{L}(x) d x\right\}\right] \\
& \pm \frac{4}{3} \delta_{L 1} e^{-2 Z r}\left[\left(\frac{3}{2} Z^{2} r^{2}+\frac{1}{2} Z r-3\right) u_{L}(r)-\left(\frac{1}{2} Z r^{2}+r\right) \frac{d u_{L}(r)}{d r}\right]
\end{aligned}
$$

where

$$
\alpha(x)=\frac{9}{2}-\frac{2}{3} e^{-2 x}\left(x^{5}+\frac{9}{2} x^{4}+9 x^{3}+\frac{27}{2} x^{2}+\frac{27}{2} x+\frac{27}{4}\right) .
$$

The scattering equation has the well-known attractive potential which for large $r$ is equal to $-\alpha /(\mathrm{Zr})^{4}, \alpha=4.5 a_{0}^{3}$ being the asymptotic polarizability for a hydrogen atom. Temkin and Lamkin [6], using this method of polarized orbitals, calculated $S$-wave phase shifts, as well as for higher partial waves, for scattering of an electron from a hydrogen atom. Phase shifts are determined by the asymptotic limit of the function $u_{L}(r)$ :

$$
\lim _{r \rightarrow \infty} u_{L}(r)=\frac{\sin \left(k r-\pi L / 2+\sigma_{C}+\eta\right)}{r} .
$$

The Coulomb phase in the above equation is given by:

$$
\sigma_{C}=\frac{Z-1}{k} \ln (2 k r)+\arg \Gamma\left(L+1-\frac{i(Z-1)}{k}\right) .
$$

$S$-wave phase shift obtained by this method are given in Table 1 and they differ from those obtained in the exchange approximation, showing the importance of polarization of the target resulting in the long-range potential $-1 / \mathrm{r}^{4}$. However, this method does not provide variational bounds to the exact phase shifts. 
The short-range correlations can be important in addition to the long-range correlations. These short-range correlations can be included using the projection-operator formalism of Feshbach [11]:

$$
P=P_{1}+P_{2}-P_{1} P_{2} . \quad \text { and } \quad Q=1-P .
$$

The wave function in Equation (1) is augmented by a correlation function $\Phi_{L}\left(\vec{r}_{1}, \vec{r}_{2}\right)$ :

$$
\Psi\left(\vec{r}_{1}, \vec{r}_{2}\right)=u\left(\vec{r}_{1}\right) \Phi_{0}\left(\vec{r}_{2}\right) \pm(1 \leftrightarrow 2)+\Phi_{L}\left(\vec{r}_{1}, \vec{r}_{2}\right)
$$

where

$$
\Phi_{L}\left(\vec{r}_{1}, \vec{r}_{2}\right)=\sum\left\{f_{L}^{\kappa, 1}\left(r_{1}, r_{2}, r_{12}\right) D_{L}^{\kappa, 1}(\theta, \phi, \psi)+f_{L}^{\kappa,-1}\left(r_{1}, r_{2}, r_{12}\right) D_{L}^{\kappa,-1}(\theta, \phi, \psi)\right\}
$$

The radial functions are of Hylleraas type and D's are rotational harmonics [12].

The projections are defined by:

$$
\begin{aligned}
& P_{1} \Phi\left(\vec{r}_{1}, \vec{r}_{2}\right)=\Phi_{0}\left(\vec{r}_{1}\right) \int \Phi_{0}\left(\vec{r}_{1}\right) \Phi_{L}\left(\vec{r}_{1}, \vec{r}_{2}\right) d \bar{r}_{1} . \\
& P_{2} \Phi\left(\vec{r}_{1}, \vec{r}_{2}\right)=\Phi_{0}\left(\vec{r}_{2}\right) \int \Phi_{0}\left(\vec{r}_{2}\right) \Phi_{L}\left(\vec{r}_{1}, \vec{r}_{2}\right) d \vec{r}_{2} .
\end{aligned}
$$

Using the projection operators $P$ and $Q$, we get the equation for the scattering function $u_{L}^{( \pm)}(r)$, having an optical potential [7]. The equation for the scattering function $u_{L}^{( \pm)}(r)$ is given by the following equation:

$$
\left.\left[-\frac{d^{2}}{d r^{2}}+\frac{L(L+1)}{r^{2}}+V_{d} \pm V_{e x}+V_{o p}^{( \pm)}(P, Q)-k^{2}\right] u_{L}^{( \pm}\right)(r)=0
$$

where $V_{d}$ and $V_{e x}$ are the direct and nonlocal exchange potentials of the 'exchange approximation' [1]. $V_{o p}^{( \pm)}(P, Q)$ is the optical potential which is negative definite and therefore attractive. The optical potential acting on $u_{L}(r)$ is given by:

$$
V_{o p}^{( \pm)}(P, Q) u_{L}=r\left\langle Y_{L 0} P H Q \frac{1}{E-Q H Q} Q H P \Psi_{L}\right\rangle
$$

We find $S$-wave phase shifts, given in Table 1, increase compared to the exchange approximation when short-range correlations are included but not the long-range correlations at the same time. However, the phase shifts obtained by this method have lower bounds to the exact phase shifts.

Is it possible to consider the long-range and short-range correlations at the same time? The projection-operator formalism, when both type of correlations are included, does not work because it is not possible to construct projection operators when $\Phi^{\text {pol }}\left(\vec{r}_{1}, \vec{r}_{2}\right)$ instead of $\Phi_{0}\left(\vec{r}_{2}\right)$ is used in the definitions given in Equation (21). However, we can write a wave function which has a long-range and a short-range parts at the same time and we call this formulation hybrid theory because it includes both type of correlations: short-range as well as long-range at the same time. We describe this formalism by confining ourselves to the e-H partial wave (denoted by $L$ ) problem. The total spatial wave function is written as:

$$
\Psi_{L}\left(\vec{r}_{1}, \vec{r}_{2}\right)=\frac{u_{L}\left(r_{1}\right)}{r_{1}} Y_{L 0}\left(\Omega_{1}\right) \Phi^{p o l}\left(\vec{r}_{1}, \vec{r}_{2}\right) \pm(1 \leftrightarrow 2)+\sum_{\lambda=1}^{N} C_{\lambda} \Phi_{L}^{\lambda}\left(\vec{r}_{1}, \vec{r}_{2}\right) .
$$

The functions $\Phi^{p o l}\left(\vec{r}_{1}, \vec{r}_{2}\right)$ and $\Phi_{L}\left(\vec{r}_{1}, \vec{r}_{2}\right)$ are given in Equations (9) and (20), respectively. This approach is variationally correct and phase shifts obtained have lower bounds to the exact phase shifts. This has been achieved by introducing separate correlation functions and then amalgamating 
them into the scattering problem, via an optical potential, in order to replace the many-particle Schrödinger equation with a single-particle Schrödinger equation.

We can derive the equation for the scattering function $\mathrm{u}(\mathrm{r})$ from (letting $\mathrm{r}_{1}=r$ ) from the functional:

$$
I=\left\langle\Psi_{L}\left(\vec{r}_{1}, \vec{r}_{2}\right)|H-E| \Psi_{L}\left(\vec{r}_{1}, \vec{r}_{2}\right)\right\rangle=0
$$

For $S$-waves, we can write:

$$
\Phi_{L=0}\left(\vec{r}_{1}, \vec{r}_{2}\right)=\sum_{l m n}^{N_{\omega}} C_{l m n}\left[e^{-\gamma r_{1}-\delta r_{2}} r_{1}^{l} r_{2}^{m} r_{12}^{n} \pm(1 \leftrightarrow 2)\right]
$$

$C_{\operatorname{lmn}}$ in Equation (26) are coefficients which are determined when eigenvalues are calculated using the Ritz variational principle. For $N_{\omega}=1$, the functional can be written as:

$$
I=A+C_{1} B+C_{1}^{2} D .
$$

where

$$
A=\left\langle\left[\Phi^{p o l} u\left(\vec{r}_{1}\right) \pm(1 \rightarrow 2)\right]|H-E|\left[\Phi^{p o l} u\left(\vec{r}_{1}\right) \pm(1 \rightarrow 2)\right]\right\rangle
$$

and

$$
\begin{aligned}
B & =2\left\langle\Phi_{L}^{1}\left(\vec{r}_{1}, \vec{r}_{2}\right)|H-E|\left[\Phi^{\text {pol }} u\left(\vec{r}_{1}\right) \pm(1 \rightarrow 2)\right]\right\rangle \\
& =4\left\langle V_{1}\left(\vec{r}_{1}\right) u\left(\vec{r}_{1}\right)\right\rangle .
\end{aligned}
$$

We can determine $C_{1}$ by:

$$
\frac{d I}{d C_{1}}=0
$$

which implies that:

$$
B+2 C_{1} D=0
$$

This gives

$$
C_{1}=-B / 2 D=\frac{2\left\langle V_{1}\left(\vec{r}_{1}\right) u\left(\vec{r}_{1}\right)\right\rangle}{E-\varepsilon_{1}} .
$$

where

$$
\begin{gathered}
\left.V_{1}\left(r_{1}\right)=\left\langle\Phi_{L}^{(1)}\left(\vec{r}_{1}, \vec{r}_{2}\right)|H-E| \Phi^{p o l}\right)\right\rangle, \\
D=\left\langle\Phi_{L}^{(1)}|H-E| \Phi_{L}^{(1)}\right\rangle=\varepsilon_{1}-E .
\end{gathered}
$$

We can generalize the above treatment to any number of unknown coefficients in Equation (24). Now all the quantities in Equation (24) are known except $u_{L}(r)$. The equation for the scattering function $u_{L}(r)$ can be derived from:

$$
\left\langle Y_{L 0}\left(\Omega_{1}\right) \Phi^{p o l}|H-E| \Psi\left(\vec{r}_{1}, \vec{r}_{2}\right)\right\rangle=0 .
$$

The resulting equation is fairly complicated and the various quantities are given in [13]. We give a few of them below. We can generalize the above treatment to any number of unknown coefficients in Equation (24). We write the wave function in the form:

$$
\Psi_{L}\left(\vec{r}_{1}, \vec{r}_{2}\right)=u_{L}\left(\vec{r}_{1}\right) \Phi^{p o l}\left(\vec{r}_{1}, \vec{r}_{2}\right) \pm(1 \leftrightarrow 2)+\sum_{\lambda} C_{\lambda} \Phi_{L}^{\lambda}\left(\vec{r}_{1}, \vec{r}_{2}\right)
$$

We define now:

$$
\Phi^{p o l}\left(\vec{r}_{1}, \vec{r}_{2}\right)=\Phi_{0}\left(\vec{r}_{2}\right)-\frac{\chi_{\beta}\left(r_{1}\right)}{r_{1}^{2}} \frac{u_{1 s \rightarrow p}\left(r_{2}\right)}{r_{2}} \frac{\cos \left(\theta_{12}\right)}{\sqrt{\pi Z}}
$$


We have replaced the step function $\varepsilon\left(r_{1}, r_{2}\right)$ by:

$$
\chi_{\beta}\left(r_{1}\right)=\left(1-e^{-\beta r_{1}}\right)^{n} .
$$

The exponent $n$ is found to be 3 or 4 . Now, polarization takes place for any value of $r_{1}$, the coordinate of the incident electron. In the method of polarized orbitals, the orbital of the target was perturbed only when the incident electron was outside the target. Now perturbation takes place whether the incident electron is outside or inside of the target.

In addition to the nonlinear parameters in the correlation function, $\beta$ is another nonlinear parameter and it is a function of the incident momentum $k$. This further helps in getting improved results. There is another form of the smooth cutoff function given by Shertzer and Temkin [14]:

$$
\chi_{S T}\left(r_{1}\right)=1-e^{-2 Z r_{1}}\left(\frac{1}{3}\left(Z r_{1}\right)^{4}+\frac{4}{3}\left(Z r_{1}\right)^{3}+2\left(Z r_{1}\right)^{2}+2 Z r_{1}+1\right) .
$$

All the quantities are now known in the wave function given in Equation (24). We can derive the equation for the scattering function using Equation (35). The equation for the scattering function is again is of the same form as Equation (22), but with more terms:

$$
\left[D(r) \frac{d^{2}}{d r^{2}}-\frac{L(L+1)}{r^{2}}+V_{d}+V_{d}^{p o l} \pm\left(V_{e x}+V_{e x}^{p o l}\right)-V_{o p}^{p o l}+k^{2}\right] u(r)=0 .
$$

As stated earlier, most of the quantities are given in [13]. Some of these quantities occurring in the direct potential are given below:

$$
D(r)=1+\frac{43}{8 Z^{6}}\left(\frac{\chi(r)}{r^{2}}\right)^{2}
$$

The direct potentials are given by:

$$
V_{d}=\frac{2(Z-1)}{r}+2 e^{-Z r}\left(Z+\frac{1}{r}\right)
$$

We repeat here the exchange terms already indicated in Equation (5):

$$
\begin{gathered}
V_{e x} u_{L}(r)=4 Z^{3} e^{-Z r}\left\{\left(k^{2}+Z^{2}\right) r \delta_{L 0} I_{1}-\frac{2}{2 L+1}\left(\frac{I_{2}}{r^{L+1}}+r^{L+1} I_{3}\right)\right\} \\
V_{d}^{p o l}=\left(x_{1}+x_{2}\right)+x_{3} \frac{d}{d r} . \\
x_{1}=\frac{43}{8 Z^{6}}\left(\frac{2 Z}{r}-\frac{2}{r^{2}}+k^{2}\right)\left(\frac{\chi(r)}{r^{2}}\right)^{2} . \\
x_{2}=B_{1}(r) \frac{43}{8 Z^{6}}\left(\frac{\chi(r)}{r^{2}}\right) . \\
x_{3}=B_{2}(r) \frac{43}{8 Z^{6}}\left(\frac{\chi(r)}{r^{2}}\right)+\frac{2 \alpha(r)}{(Z r)^{4}} \chi(r)-\frac{9}{2} \frac{\chi(r)^{2}}{(Z r)^{4}}-\frac{8}{3 Z} d(r)\left(\frac{\chi(r)}{r^{2}}\right)^{2}, \\
B_{2}(r)=-2 Z B_{1}(r)-\frac{1}{r^{2}}\left[-2 Z \chi(r)+2 Z-\frac{2}{r}+e^{-2 Z r}\left(-\frac{2}{3} Z^{4} r^{3}-\frac{4}{3} Z^{3} r^{2}+2 Z+\frac{2}{r}\right)\right], \\
\alpha(r)=\frac{9}{2}-\frac{2}{3} e^{-2 Z r}\left(\frac{3}{2}(Z r)^{4}+\frac{15}{2}\left(Z Z^{5} r+2 Z^{4}-\frac{4 Z^{2}}{r^{2}}-\frac{8 Z}{r^{3}}-\frac{6}{r^{4}}\right) .\right.
\end{gathered}
$$


The last term in $x_{3}$ has:

$$
\begin{gathered}
d(r)=\frac{129}{32 Z^{5}} \frac{1}{r}+\frac{18}{Z^{7}} \frac{1}{r^{3}}-e^{-2 Z r}\left(\frac{3}{16} r^{4}+\frac{27}{16 Z} r^{3}+\frac{54}{8 Z^{2}} r^{2}+\frac{135}{8 Z^{3}} r\right. \\
\left.+\frac{975}{32 Z^{4}}+\frac{1281}{32 Z^{5}} \frac{1}{r}+\frac{36}{Z^{6}} \frac{1}{r^{2}}+\frac{18}{Z^{7}} \frac{1}{r^{3}}\right)
\end{gathered}
$$

For $r \rightarrow \infty, x_{3}$ has a term

$$
\frac{9}{2 Z^{4}}
$$

which is the dipole polarizability of the target with nuclear charge $Z$. $V_{o p}^{p o l}=$ optical potential which is attractive, but does not depend on projection operators, and is given by:

$$
V_{o p}^{p o l} u_{L}\left(r_{1}\right)=r_{1} \sum_{s}^{N_{\omega}} \frac{\left.\left\langle Y_{L 0} \Omega_{1}\right) \Phi^{p o l}\left(\vec{r}_{1}, \vec{r}_{2}\right)|H-E| \Phi_{L}^{s}\right\rangle\left\langle\Phi_{L}^{s}|H-E| \Psi_{L}^{\prime}\right\rangle}{E-\varepsilon_{s}} .
$$

The function $\Psi_{L}^{\prime}$ is the function given in Equation (24) without the correlation terms. Phase shifts, given in Table 2, are calculated using the asymptotic limit of the function $u_{L}(r)$ given in Equation (16).

We see that the method of polarized orbitals over estimates phase shifts. In the variational formulation, phase shifts have lower bounds to the exact phase shifts, that is, they can be higher than those given in Table 2 when the number of correlations terms in Equation (24) is increased. The phase shifts obtained using hybrid theory agree well with those obtained by Schwartz [3] and also with those obtained by Scholz et al. [15] using the close-coupling approximation. Similar calculations have been carried out for $S$-wave electron scattering from $\mathrm{He}^{+}$and $\mathrm{Li}^{2+}[16]$. These results are shown in Table 3 and they agree with those obtained by Gien $[17,18]$ using the Harris-Nesbet variational method.

Calculations have been carried out for partial wave $L=1$ for electron scattering from $\mathrm{H}, \mathrm{He}^{+}$, and $\mathrm{Li}^{2+}[19,20]$. The $P$-wave phase shifts for electron-H scattering are given in Table 4 . They are compared with those obtained using the $R$-matrix method [15] and by the finite-element method [21]. We see that the singlet $P$ phase shifts have a peak at $k=0.3$, while the triplet $P$ phase shifts increase continuously.

Table 2. Comparison of phase shifts (radians) obtained in hybrid theory [13] with those obtained by Schwartz [3] and Scholz et al. [15], the results for $k=0$ are scattering lengths.

\begin{tabular}{ccccccc}
\hline \multirow{2}{*}{$k$} & \multicolumn{3}{c}{ Singlet } & \multicolumn{3}{c}{ Triplet } \\
\cline { 2 - 7 } & $\eta_{H B}[13]$ & $\eta_{\text {schwartz }}[3]$ & $\eta_{\text {SSB }}[15]$ & $\eta_{H B}[13]$ & $\eta_{\text {Schwartz }}[3]$ & $\eta_{S S B}[15]$ \\
\hline 0.0 & 5.99567 & 5.965 & & 1.78154 & 1.7686 & \\
0.1 & 2.55370 & 2.553 & 2.550 & 2.93856 & 2.9388 & 2.939 \\
0.2 & 2.06717 & 2.0673 & 2.062 & 2.71751 & 2.7171 & 2.717 \\
0.3 & 1.69684 & 1.6964 & 1.691 & 2.49987 & 2.4996 & 2.500 \\
0.4 & 1.41554 & 1.4146 & 1.410 & 2.29457 & 2.2938 & 2.294 \\
0.5 & 1.20195 & 1.202 & 1.196 & 2.10574 & 2.1046 & 2.105 \\
0.6 & 1.04191 & 1.041 & 1.035 & 1.93336 & 1.9329 & 1.933 \\
0.7 & 0.93084 & 0.930 & 0.925 & 1.77999 & 1.7797 & 1.780 \\
0.8 & 0.88802 & 0.886 & & 1.64444 & 1.643 & \\
\hline
\end{tabular}


Table 3. Phase shifts (radians) for scattering of electrons from $\mathrm{He}^{+}$and $\mathrm{Li}^{2+}[16]$.

\begin{tabular}{ccccc}
\hline \multirow{2}{*}{$k$} & \multicolumn{2}{c}{$\mathbf{H e}^{+}$} & \multicolumn{2}{c}{$\mathbf{L i}^{\mathbf{2 +}}$} \\
\cline { 2 - 5 } & ${ }^{\mathbf{1}} \mathbf{S}$ & ${ }^{3} \mathbf{S}$ & ${ }^{\mathbf{S}}$ & ${ }^{3} \mathbf{S}$ \\
\hline 0.1 & 0.43808 & 0.93065 & 0.23188 & 0.56084 \\
0.2 & 0.43550 & 0.92704 & 0.23176 & 0.56020 \\
0.3 & 0.43142 & 0.92114 & 0.23148 & 0.55869 \\
0.4 & 0.42608 & 0.91302 & 0.23109 & 0.55678 \\
0.5 & 0.41974 & 0.90282 & 0.23064 & 0.55435 \\
0.6 & 0.41265 & 0.89057 & 0.23012 & 0.55142 \\
0.7 & 0.40568 & 0.87645 & 0.22960 & 0.54799 \\
0.8 & 0.39865 & 0.86066 & 0.22906 & 0.54413 \\
0.9 & 0.39123 & 0.84366 & $0 / 22855$ & 0.53925 \\
1.0 & 0.38644 & 0.82536 & 0.22807 & 0.53514 \\
1.1 & 0.38200 & 0.80636 & 0.22769 & 0.53000 \\
1.2 & 0.37914 & 0.78677 & 0.22740 & 0.52456 \\
1.3 & 0.37846 & 0.76696 & 0.22724 & 0.51880 \\
1.4 & 0.38158 & 0.74708 & 0.22724 & 0.51276 \\
1.5 & 0.39802 & 0.72746 & 0.22742 & 0.50646 \\
1.6 & 0.34480 & 0.70815 & 0.22782 & 049997 \\
\hline
\end{tabular}

Table 4. $P$-wave phase shifts (radians) for electron-hydrogen scattering. $\eta_{H B}$ are the results obtained using hybrid theory [19], $\eta_{R M}$ are the $R$-matrix results [15], and $\eta_{F E M}$ are finite-element method results [21].

\begin{tabular}{ccccccc}
\hline \multirow{2}{*}{$\boldsymbol{K}$} & \multicolumn{3}{c}{${ }^{\mathbf{P}}$} & \multicolumn{3}{c}{${ }^{3} \boldsymbol{P}$} \\
\cline { 2 - 7 } & $\eta_{H B}$ & $\eta_{R M}$ & $\eta_{\text {FEM }}$ & $\eta_{H B}$ & $\eta_{R M}$ & $\eta_{F E M}$ \\
\hline 0.1 & 0.00635076 & 0.006 & 0.006 & 0.01038234 & 0.010 & 0.0100 \\
0.2 & 0.01506556 & 0.015 & 0.0148 & 0.04536735 & 0.045 & 0.0452 \\
0.3 & 0.01670634 & 0.016 & 0.0160 & 0.1069312 & 0.107 & 0.1067 \\
0.4 & 0.01015347 & 0.009 & 0.0090 & 0.1888873 & 0.187 & 0.1873 \\
0.5 & -0.00061223 & -0.002 & -0.0020 & 0.2709762 & 0.270 & 0.2708 \\
0.6 & -0.01009367 & -0.012 & -0.0117 & 0.3416749 & 0.341 & 0.3417 \\
0.7 & -0.01321557 & -0.016 & -0.0149 & 0.3932100 & 0.392 & 0.3933 \\
0.8 & -0.0046818 & & -0.0068 & 0.4277296 & & 0.4283 \\
\hline
\end{tabular}

Calculations have also been carried for partial wave $L=2$ for electron scattering from $\mathrm{H}, \mathrm{He}^{+}$, and $\mathrm{Li}^{2+}$ and photoabsorption when the final state is a $2 p$ state [22].

\section{Scattering Length}

The scattering length $a$ (in units of Bohr radius $a_{0}$ ) is defined by:

$$
\lim _{k \rightarrow 0} k \cot (\eta)=-1 / a
$$

Temkin [23] has shown that the scattering length is significantly affected by the long-range polarization potential. It is given by:

$$
a=a(R)-\alpha\left(\frac{1}{R}-\frac{a}{R^{2}}+\frac{a^{2}}{3 R^{3}}\right)
$$

where $\alpha$ is the fine structure constant.

For ${ }^{1} S$, for $N_{\omega}=70$ we find $a(\mathrm{R})=6.00239$ at $R=117.3038$, using the above correction, we get $a=5.96595$ compared to the value $5.965 \pm 0.0003$ obtained by Schwartz [3] who used the Kohn variational principle. The ${ }^{3} S$ scattering length for $N_{\omega}=84$ is $a(R)=1.781542$ at $R=349.0831$. The corrected value is 1.76815 is little lower compared to the Schwartz $a=1.7686 \pm 0.0002$. The agreement with Schwartz's values for both singlet and triplet $S$ scattering lengths is very good.

The hybrid theory is akin to the close-coupling approximation in which the wave function is expanded in the orthonormal eigenstates of the target $1 s, 2 s, 2 p \ldots$. However, in the close-coupling 
approximation, the asymptotic polarizability of the target depends on the number of $p$ states included in the wave function. If $1 s, 2 s$, and $2 p$ eigenstates are included, then $66 \%$ of the polarizability is included, while in the hybrid theory the total asymptotic polarizability of the target is included and these calculations are also variationally correct. Also, the convergence in the close-coupling approximation is very slow as the number of eigenstates is increased.

\section{Resonances}

Calculations for phase shifts have been carried out for $\mathrm{He}^{+}$and $\mathrm{Li}^{+2}$ targets in the resonance region [16]. These phase shifts are fitted to the Breit-Wigner form to obtain the resonance parameters:

$$
\eta_{\text {calc. }}(E)=\eta_{0}+A E+\tan ^{-1}\left(\frac{0.5 \Gamma}{\left(E_{R}-E\right)}\right) .
$$

$E=k^{2}$ is the incident energy, $E_{R}$ is the resonance position and $\Gamma$ is its width. The present calculation gives [16] $E_{R}=57.8481$ and $\Gamma=0.1233 \mathrm{eV}$ for ${ }^{1} S$ state of He. The resonance position is with respect to the ground state of the He atom. These results agree well with those obtained using the Feshbach formalism [24]:

$$
E_{R}=57.8435 \text { and } \Gamma=0.125 \mathrm{eV} \text {. }
$$

In the Feshbach formalism, $E_{R}=\varepsilon_{Q}+\Delta$, where $\varepsilon_{Q}=\langle Q H Q\rangle$ and $\Delta$ is the correction due to the fact that the resonance is embedded in the continuum and there are other resonance states nearby. These corrections have to be calculated separately in order to get the position given above, as indicated in [24]. There are no such corrections when the resonance position is obtained by fitting resonance-region phase shifts to the Breit-Wigner expression given in Equation (55). There is no bound on the resonance position because of fitting of the phase shifts to $E_{R}$ and $\Gamma$ in Equation (55) even when there is a lower bound on the phase shifts.

\section{Low-Energy Scattering}

It is known [25] that at low energies $L=1$ scattering, the long-range correlations contribute most to the scattering phase shifts:

$$
\tan (\eta) / k^{2}=\pi \alpha / 15-A k
$$

So that

$$
\tan \eta\left(k_{1}\right) / k_{1}^{2}-\tan \eta\left(k_{2}\right) / k_{2}^{2}=-A\left(k_{1}-k_{2}\right) .
$$

The first term in Equation (57) is due to the long-range potential ( $\alpha$ is the polarizability) and the second term has contributions from the short-range correlations. Using the phase shifts given in Table 5, we find:

$$
A^{T}=-1.002 \text { and } A^{S}=2.942 \text {. }
$$

Table 5. Low-energy $P$-wave phase shifts for electron-hydrogen scattering.

\begin{tabular}{ccc}
\hline State & $\boldsymbol{k}_{\mathbf{1}}=\mathbf{0 . 0 4}$ & $\boldsymbol{k}_{\mathbf{2}}=\mathbf{0 . 0 5}$ \\
\hline${ }^{1} P$ & 0.001303692 & 0.001963464 \\
${ }^{3} P$ & 0.001564346 & 0.002469346 \\
\hline
\end{tabular}

These value compare reasonably well with the results $A^{T}=-1.3$ and $A^{S}=1.6$ obtained in [25] by using the phase shifts obtained by the method of polarized orbitals [6]. The results in [6] are not accurate enough because of lack of any variational principle which is the reason for discrepancy.

For $L$ equal to 1 and greater than 1 , the first term in the expansion of $\tan \eta(k)$ is always proportional $k^{2}$ as given below:

$$
\tan \eta(k)=\frac{\pi \alpha k^{2}}{(2 L-1)(2 L+1)(2 L+3)}-A k^{2 L+1} .
$$


For $L=0$, the expansion is complicated and involves terms like $k, k^{2}, \ln (k)$, and the effective range. It is given in Equation (49) of reference [25].

\section{Photoabsorption}

It was pointed out by Wildt [26] that the opacity in the sun is due to the absorption of photons:

$$
\begin{aligned}
& h v+H^{-}=H+e, \\
& h v+H e=H e^{+}+e .
\end{aligned}
$$

The photoabsorption or photodetachment cross section (in length form and in units of $\left(a_{0}^{2}\right)$ for a transition from an initial state ' $i$ ' to the final state ' $f$ ' is given by:

$$
\sigma=4 \pi \alpha k \omega\left|\left\langle\Psi_{f}\left|z_{1}+z_{2}\right| \Phi_{i}\right\rangle\right|^{2},
$$

where $\alpha$ is the fine structure constant, $k$ is the momentum of the outgoing electron, and $\omega$ is the energy of the incident photon:

$$
\omega=I+k^{2},
$$

where $I$ is the ionization of the system absorbing the photon and $k^{2}$ is the energy of the ejected electron. The Hylleraas-type normalized function $\Phi_{i}$ is the ground-state wave function of the two-electron systems and it is given by:

$$
\Phi_{i}=\frac{1}{\sqrt{8 \pi^{2}}} \sum C_{l m n}\left[e^{-a r_{1}-b r_{2}} r_{1}^{l} r_{2}^{m} \pm(1 \leftrightarrow 2)\right] r_{12}^{n} .
$$

The upper sign refers to the singlet states and the lower sign refers to the triplet states. In the Table 6, the photodetachment cross sections of $H^{-}$[27] are given for a few values of $k$, momentum of the ejected electron. These results have been obtained without the short-range correlations. They are compared in Table 6 with those obtained by Bell and Kingston [28] who used the method of polarized orbitals. Their results are higher than those obtained using the variational approach [27]. Addition of short-range correlations in the final state wave function gave improved results for cross sections, given in Table 7. However, we find that at low energies the cross sections do not change considerably, showing that at low-energies the long-range correlations are more important than the short-range correlations.

Table 6. Photodetachment cross sections $(\mathrm{Mb})$ of the ground state of $\mathrm{H}^{-}$, without short-range correlations [27] and comparison with those obtained by Bell and Kingston [28].

\begin{tabular}{ccccc}
\hline $\mathbf{K}$ & $\boldsymbol{N}_{\boldsymbol{\omega}}=\mathbf{2 2 0}$ & $\mathbf{2 8 6}$ & $\mathbf{3 6 4}$ & Ref. [28] \\
\hline 0.01 & 0.0243 & 0.244 & 0.0245 & \\
0.05 & 2.7015 & 2.7148 & 2.7480 & \\
0.1 & 15.2147 & 15.2324 & 15.2465 & 12.34 \\
0.2 & 38.3763 & 38.3675 & 38.3688 & 40.48 \\
0.3 & 34.9783 & 34.9806 & 34.9684 & 36.40 \\
0.4 & 24.2498 & 24.2472 & 24.2537 & 25.296 \\
0.5 & 15.8663 & 15.8699 & 15.8692 & 16.43 \\
0.6 & 10.4972 & 10.4942 & 10.4924 & 11.29 \\
0.7 & 7.1234 & 7.1243 & 7.1258 & \\
0.74 & 6.1508 & 6.1521 & 6.1530 & \\
0.8 & 4.9762 & 4.9769 & 4.9768 & 5.31 \\
0.8544 & 4.1423 & 4.1425 & 4.1421 & \\
0.8631 & 4.0230 & 4.0228 & 4.0224 & \\
0.8660 & 3.9852 & 3.9851 & 3.9846 & \\
\hline
\end{tabular}


Table 7. Photodetachment cross sections $(\mathrm{Mb})$ of the ground state of $\mathrm{H}^{-}$, with short-range correlations included in the final state [27].

\begin{tabular}{cccc}
\hline $\mathbf{K}$ & $\boldsymbol{N}_{\boldsymbol{\omega}}=\mathbf{2 2 0}$ & $\mathbf{2 8 6}$ & $\mathbf{3 6 4}$ \\
\hline 0.04 & 1.4464 & 1.4545 & 1.4750 \\
0.05 & 2.7050 & 2.7185 & 2.7517 \\
0.1 & 15.2526 & 15.2704 & 15.3024 \\
0.2 & 38.5516 & 38.5429 & 38.5443 \\
0.23 & 39.5764 & 39.5927 & 39.6366 \\
0.25 & 39.0699 & 39.0925 & 39.1350 \\
0.3 & 35.2420 & 35.2443 & 35.2318 \\
0.4 & 24.4734 & 24.4709 & 24.4774 \\
0.5 & 16.0830 & 16.0866 & 16.0858 \\
0.6 & 10.7459 & 10.7428 & 10.7410 \\
0.7 & 7.4837 & 7.4847 & 7.4862 \\
0.74 & 6.6050 & 6.6063 & 6.6072 \\
0.800 & 5.6506 & 5.6514 & 5.6512 \\
0.8544 & 4.1426 & 4.1425 & 4.1421 \\
0.8631 & 6.8980 & 6.8984 & 6.8976 \\
0.8660 & 7.6229 & 7.6230 & 7.6223 \\
\hline
\end{tabular}

Ohmura and Ohmura [29], using the effective range theory and the loosely bound structure of hydrogen ion, obtained:

$$
\sigma=\frac{6.8475 \times 10^{-18} \gamma k^{3}}{(1-\gamma \rho)\left(\gamma^{2}+k^{2}\right)^{3}} \mathrm{~cm}^{2} .
$$

where $\gamma=0.2355883$ and $\rho=2.646 \pm 0.0004, \gamma$ is the square root of the binding energy of the electron and $\rho$ is the effective range. The cross sections obtained using the effective-range theory are higher than those obtained in [27]. However, at low energies they are close to each other. Photodetachment cross sections for $\mathrm{H}^{-}$are shown in Figure 1 from Ref. [27].

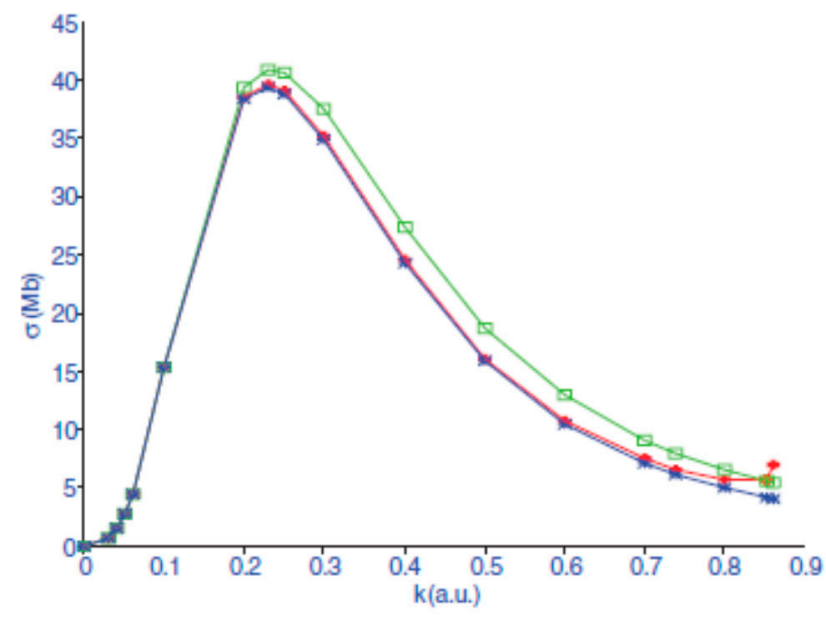

Figure 1. Photodetachment of detachment of a hydrogen ion. The lowest curve is obtained only when the long-range correlations are included; the middle curve is obtained when the short-range and long-range correlations are included. The top [27] curve is obtained when the effective range theory and the loosely bound structure of hydrogen ion used [29].

A similar calculation, including short-range and long-range correlations, has been carried out for the photoionization of He [27]. The results, given in Table 8, agree well with those obtained using the $R$-matrix approach [30] and the experimental results of Samson et al. [31]. Photoionization cross sections for Li ion have also been calculated and they are also given in [27]. 
Table 8. Photoionization cross sections $(\mathrm{Mb})$ of He [27] obtained with correlations.

\begin{tabular}{cccccc}
\hline $\mathbf{k}$ & $\boldsymbol{N}_{\mathbf{!}}=\mathbf{1 2 0}$ & $\mathbf{1 6 5}$ & $\mathbf{2 2 0}$ & Ref. [30] & Ref. [31] \\
\hline 0.1 & 7.3319 & 7.3305 & 7.3300 & 7.295 & 7.44 \\
0.2 & 7.1563 & 7.1549 & 7.1544 & 7.115 & 7.13 \\
0.3 & 6.8733 & 6.8720 & 6.8716 & 6.838 & 6.83 \\
0.4 & 6.4965 & 6.4953 & 6.4951 & 6.474 & 6.46 \\
0.5 & 6.0471 & 6.0461 & 6.0461 & 6.006 & 6.02 \\
0.6 & 5.5929 & 5.5924 & 5.5925 & 5.535 & 5.55 \\
0.7 & 5.0121 & 5.0118 & 5.0120 & 4.985 & 5.04 \\
0.8 & 4.4738 & 4.4738 & 4.4740 & 4.482 & 4.51 \\
0.9 & 3.9647 & 3.9648 & 3.9649 & & \\
1.0 & 3.4652 & 3.4654 & 3.4654 & 3.476 & 3.48 \\
1.1 & 3.0205 & 3.0206 & 3.0206 & 3.023 & 3.00 \\
1.3 & 2.2560 & 2.2561 & 2.2561 & 2.271 & 2.19 \\
1.4 & 1.9820 & 1.9821 & 1.9821 & 1.943 & 1.89 \\
1.5 & 1.6816 & 1.6817 & 1.6817 & & \\
1.6 & 1.6324 & 1.6329 & 1.6329 & & \\
\hline
\end{tabular}

We notice that in the limit $k \rightarrow 0$, the photodetachment cross section for $\mathrm{H}^{-}$goes to zero because the plane wave is normalized as $\sin (k r) / k r$ which is equal to 1 for $r \rightarrow 0$, while the photoionization cross section of He goes to a finite value because of the final state Coulomb wave, which is proportional to $\frac{1}{\sqrt{k}}$ for $r \rightarrow 0$.

\section{Recombination Rates}

Knowing the photoabsorption cross sections, we can calculate radiative attachment rate coefficients which are important in the solar and astrophysical problems.

$$
\begin{aligned}
& e+H \rightarrow H^{-}+h v \\
& e+\mathrm{He}^{+} \rightarrow \mathrm{He}+h v
\end{aligned}
$$

The attachment cross section $\sigma_{a}$ is given by:

$$
o_{a}=\left(\frac{h v}{c}\right)^{2} \frac{1}{2 m E} \frac{g(f)}{g(i)} \sigma .
$$

This relation follows from the principle of the detailed balance. The weight factors in the initial and final states are $g(i)$ and $g(f), E=k^{2}$ is the energy of the electron, and $k_{B}$ is the Boltzmann constant in Equation (66), which gives the rate coefficient:

$$
\alpha_{R}(T)=\frac{(2 S+1) 10.2509 \times 10^{10}}{(T)^{1.5}} \int_{0}^{\infty} d E(E+I)^{2} \sigma e^{-E / k_{B} T} .
$$

In Table 9, the rate coefficients for the singlet states of $\mathrm{H}^{-}, \mathrm{He}$, and $\mathrm{Li}^{+}$are given at various temperatures [27]. We see that the rate coefficients for $\mathrm{H}^{-}$and $\mathrm{Li}^{+}$ions increase with temperature, attain a maximum value and then decrease, while in He atoms they decrease monotonically with the increase of the temperature. 
Table 9. Recombination coefficients $\left(\mathrm{cm}^{3} / \mathrm{s}\right)$ for singlet states of $\mathrm{H}^{-}, \mathrm{He}$, and $\mathrm{Li}^{+}$[27].

\begin{tabular}{|c|c|c|c|}
\hline$T$ & $\alpha_{R}(T) \times 10^{15}, \mathrm{H}^{-}$ & $\alpha_{R}(T) \times 10^{13}, \mathrm{He}$ & $\alpha_{R}(T) \times 10^{13}, \mathrm{Li}^{+}$ \\
\hline 1000 & 0.99 & 2.50 & 0.12 \\
\hline 2000 & 1.28 & 2.39 & 1.04 \\
\hline 5000 & 2.40 & 1.87 & 2.62 \\
\hline 7000 & 2.82 & 1.66 & 2.92 \\
\hline 10,000 & 3.20 & 1.45 & 3.03 \\
\hline 12,000 & 3.37 & 1.35 & 3.02 \\
\hline 15,000 & 3.56 & 1.23 & 2.95 \\
\hline 17,000 & 3.65 & 1.17 & 2.89 \\
\hline 20,000 & 3.75 & 1.10 & 2.79 \\
\hline 22,000 & 3.79 & 1.05 & 2.73 \\
\hline 250,000 & 3.83 & 0.99 & 2.63 \\
\hline 30,000 & 3.83 & 0.92 & 2.49 \\
\hline 35,000 & 3.77 & 0.87 & 2.36 \\
\hline 40,000 & 3.67 & 0.82 & 2.25 \\
\hline
\end{tabular}

Photoabsorption cross sections for excited states of the above systems and the recombination rate coefficients associated with these states have also been calculated [27]. In Table 10, rate coefficients for the excited states of $\mathrm{He}$ atoms and $\mathrm{Li}^{+}$ions are given. As is well known, there are no excited states of $\mathrm{H}^{-}$, therefore no results are given for this ion. We see that the singlet and triplet states the rate coefficients differ quite a bit.

Table 10. Rate coefficients $\left(\mathrm{cm}^{3} / \mathrm{s}\right)$ for $(1 \mathrm{~s} 2 \mathrm{~s})^{3} \mathrm{~S}$ and $(1 \mathrm{~s} 2 \mathrm{~s}) 1 \mathrm{~S}$ singlet excited states of He atom and $\mathrm{Li}^{+}$ ion [27].

\begin{tabular}{|c|c|c|c|c|}
\hline \multirow{2}{*}{$T$} & \multicolumn{2}{|c|}{$\mathrm{He}$} & \multicolumn{2}{|c|}{$\mathrm{Li}^{+}$} \\
\hline & $\alpha_{R}(T) \times 10^{14},{ }^{3} S$ & $\alpha_{R}(T) \times 10^{15},{ }^{1} S$ & $\alpha_{R}(T) \times 10^{14},{ }^{3} S$ & $\alpha_{R}(T) \times 10^{14},{ }^{1} S$ \\
\hline 1000 & 2.13 & 8.27 & 4.68 & 2.99 \\
\hline 2000 & 2.08 & 7.97 & 4.47 & 2.87 \\
\hline 5000 & 1.73 & 7.30 & 3.48 & 2.27 \\
\hline 7000 & 1.56 & 5.71 & 3.09 & 2.03 \\
\hline 10,000 & 1.40 & 5.05 & 2.68 & 1.78 \\
\hline 12,000 & 1.32 & 4.73 & 2.49 & 1.66 \\
\hline 15,000 & 1.23 & 4.35 & 2.26 & 1.52 \\
\hline 17,000 & 1.18 & 4.15 & 2.14 & 1.45 \\
\hline 20,000 & 1.12 & 3.90 & 1.98 & 1.36 \\
\hline 22,000 & 1.09 & 3.75 & 1.90 & 1.31 \\
\hline 25,000 & 1.04 & 3.57 & 1.79 & 1.24 \\
\hline 30,000 & 0.98 & 3.31 & 1.64 & 1.15 \\
\hline 35,000 & 0.93 & 3.10 & 1.52 & 1.08 \\
\hline 40,000 & 0.89 & 2.93 & 1.43 & 1.02 \\
\hline
\end{tabular}

\section{Excitation of the $2 S$ State}

Cross section for excitation of the $2 S$ state of atomic hydrogen at low incident electron energies has been carried out using different methods. We mention a few: Burke et al. [32] used close-coupling approximation retaining $1 s, 2 s$, and $2 p$ eigenstates. Callaway et al. [33] carried out calculations in the range 12 to $54 \mathrm{eV}$ using 11-state expansion including seven pseudostates. A similar calculation in the above energy range has been carried by Callaway et al. [34] using six-state close-coupling expansion including an optical potential. The results of these calculations are essentially based on the close-coupling approximation. Scott el al. [35] used the standard $R$-matrix close-coupling method using nine-state basis, consisting of three eigenstates and six pseudostates. Poet [36] carried out calculations by assuming spherically symmetric wave functions. Lloyd and McDowell [37] used the method of polarized orbitals for the first three partial waves and Bessel functions for the higher partial waves. 
All these results are different from each other. Even though the hydrogen atom is the simplest system, it seems it is not easy to get exact cross sections for excitation. Excitation cross sections have been calculated using the variational method of polarized orbitals [13]. The excitation cross section [38] from state ' $i$ ' to state ' $f$ ' is given by:

$$
\sigma=\frac{k_{f}}{k_{i}} \int\left|T_{f i}\right|^{2} d \Omega
$$

where $k_{i}$ and $k_{f}$ are the initial and final momenta and $T_{f i}$ is a matrix element given by:

$$
T_{f i}=-(1 / 4 \pi)\left\langle\psi_{f}|V| \Psi_{i}\right\rangle .
$$

The interaction potential is:

$$
V=-\frac{2 Z}{r_{1}}+\frac{2}{r_{12}}
$$

The initial state wave function $\Psi_{i}$, in principle, is an exact solution of the Schrödinger equation [38]. Using this method, excitation cross sections have been calculated in the energy range 10.30 to $54.5 \mathrm{eV}$ by using nine partial waves to get convergence, which is good up to the fifth and sixth decimal places for low energies, while at high energies it is good up to the third decimal place [38]. Some of the results of the various calculations are given in Table 11. The maximum of the cross section $0.137 \pi a_{0}^{2}$ at $11.4 \mathrm{eV}$ which is close to the experimental result $0.163 \pm 0.2 \pi a_{0}^{2}$ at $11.6 \pm 0.2 \mathrm{eV}$ of Kauppila et al. [39]. There is a minimum of the cross section at $16.46 \mathrm{eV}$ and another maximum at $34.82 \mathrm{eV}$, as shown in Figure 2 from Ref. [38]. Differential and spin cross sections have also been calculated [38].

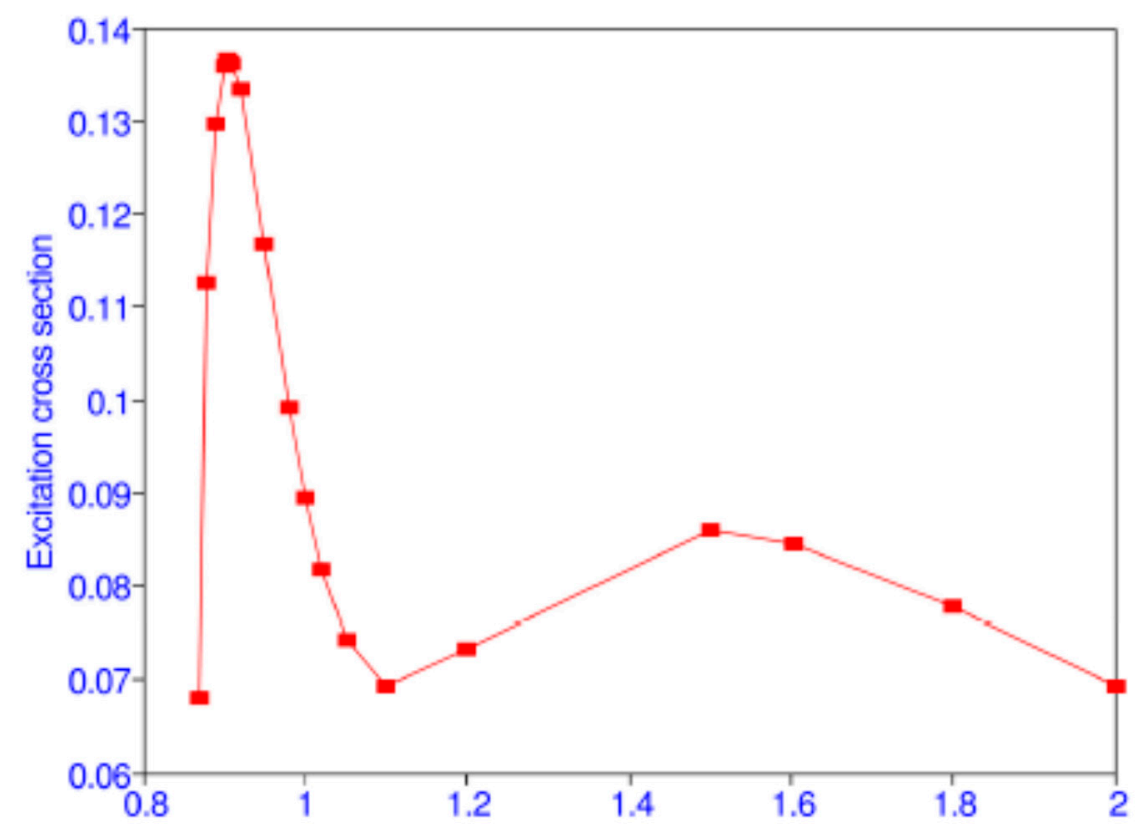

Figure 2. (Color online) Total $1 S-2 S$ excitation cross section $\left(\pi a_{0}^{2}\right)$ vs. the incident momentum $k(\operatorname{Ryd})$. 
Table 11. Excitation cross sections $\left(\pi a_{0}^{2}\right)$ obtained in using variational polarized orbital method [40] and comparison with the results of other calculations.

\begin{tabular}{ccccc}
\hline $\mathbf{K}$ & Hybrid Theory [38] & Lloyd and McDowell [37] & Burke et al. [32] & Callaway [33] \\
\hline 0.87 & $6.8208(-2)$ & 0.1789 & & \\
0.90 & $1.3589(-1)$ & & 0.09918 & \\
0.904 & $1.3667(-1)$ & & & \\
0.905 & $1.3673(-1)$ & & & \\
0.906 & $1.3662(-1)$ & & & \\
0.907 & $1.3660(-1)$ & 0.1443 & 0.1783 & 0.161 \\
0.98 & $9.9106(-2)$ & & & 0.154 \\
1.00 & $8.9474(-2)$ & & & 0.150 \\
1.02 & $8.1852(-2)$ & & 0.1654 & 0.142 \\
1.05 & $8.4057(-2)$ & & 0.1349 & 0.135 \\
1.10 & $6.9444(-2)$ & & 0.0891 & 0.111 \\
1.20 & $7.3317(-2)$ & & & 0.087 \\
1.50 & $8.5797(-2)$ & & & 0.081 \\
1.60 & $8.4611(-2)$ & 0.1198 & 0.06355 & 0.074 \\
1.80 & $7.1804(-2)$ & & & 0.066 \\
2.00 & $6.9460(-2)$ & & & \\
\hline
\end{tabular}

\section{Lyman-Alpha Radiation}

When the transition $(2 p->1 s)$ takes place, we get electric dipole Lyman-alpha radiation which has a wavelength of $1216 \mathrm{~A}^{0}$ in $\mathrm{H}$ atoms and $304 \mathrm{~A}^{0}$ in He atoms. This radiation has been seen from the sun and from various astrophysical sources, and also from the Milky Way galaxy [41]. In most calculations, the $2 p$ state is excited by electron impact. However, photoexciation is also possible: instead of leaving the target in the $1 s$ state, it is left in the $2 p$ state. The outgoing electron can then be in the final state $l_{f}=0$ or 2 . In the dipole approximation, the cross section is:

$$
\sigma=\frac{4 \pi \alpha k \omega}{3\left(2 l_{i}+1\right)}\left(\left|M_{0}\right|^{2}+\left|M_{2}\right|^{2}\right)
$$

The matrix element $M$ is defined as:

$$
M=\left(2 l_{f}+1\right)^{0.5}\left|\left\langle\Psi_{f}\left|z_{1}+z_{2}\right| \Phi_{i}\right\rangle\right| .
$$

In Table 12, we give photoabsorption cross sections [40] for two-electron systems for various values of the outgoing electron leaving the photoionized target in $2 p^{2} P$ state. The calculation in this problem has been carried out using only the exchange approximation for the final continuum states. No short-range and long-range correlations have been included in this calculation. The purpose was to show that the cross sections are significant and this process should be considered along with excitation by electron impact. Photoabsorption cross sections when the final state is $2 s^{2} S$ instead of $2 p^{2} P$ are also given in [40], along with the recombination rate coefficients. 
Table 12. Photoabsorption cross sections $(\mathrm{Mb})$ of the $\mathrm{H}$ anion, $\mathrm{He}, \mathrm{Li}^{+}, \mathrm{Be}^{2+}$, and $\mathrm{C}^{4+}$. The final state is the ${ }^{2} P$ state $[40]$.

\begin{tabular}{cccccc}
\hline $\mathbf{K}$ & $\mathbf{H}^{-}$ & $\mathbf{H e}$ & $\mathbf{L i}^{+}$ & $\mathbf{B e}^{2+}$ & $\mathbf{C}^{4+}$ \\
\hline 0.1 & 1.2511 & $3.4706(-2)$ & & & \\
0.2 & 1.6983 & $2.2972(-2)$ & $3.8740(-3)$ & $6.9296(-3)$ & $1.8170(-4)$ \\
0.3 & 1.6578 & $3.0459(-2)$ & $3.8183(-3)$ & $6.7374(-3)$ & $1.8144(-4)$ \\
0.4 & 1.1709 & $2.7627(-2)$ & $3.7482(-3)$ & $6.4820(-3)$ & $1.8104(-4)$ \\
0.5 & $6.4369(-1)$ & $2.4948(-2)$ & $3.6694(-3)$ & $6.1657(-3)$ & $1.8048(-4)$ \\
0.6 & $4.4867(-1)$ & $2.2717(-2)$ & $3.5894(-3)$ & $5.8193(-3)$ & $1.7987(-4)$ \\
0.7 & $4.1448(-1)$ & $2.1120(-2)$ & $3.5102(-3)$ & $5.4450(-3)$ & $1.7916(-4)$ \\
0.8 & $3.5327(-1)$ & $2.0058(-2)$ & $3.4337(-3)$ & $5.0471(-3)$ & $1.7836(-4)$ \\
0.9 & $2.6847(-1)$ & $1.9375(-2)$ & $3.3712(-3)$ & $4.6498(-3)$ & $1.7505(-4)$ \\
1.0 & $1.508(-1)$ & $1.8811(-2)$ & $3.3083(-3)$ & $4.2507(-3)$ & $1.7641(-4)$ \\
1.1 & & $1.8219(-2)$ & $3.2481(-3)$ & $3.8543(-3)$ & $1.7552(-4)$ \\
1.2 & & $1.7409(-2)$ & $3.1894(-3)$ & $3.4825(-3)$ & $1.7437(-4)$ \\
1.3 & & $1.6392(-2)$ & $3.1220(-3)$ & $3.1270(-3)$ & $1.7322(-4)$ \\
1.4 & & $1.5181(-2)$ & $3.0533(-3)$ & $2.8077(-3)$ & $1.7198(-4)$ \\
1.5 & & $1.3866(-2)$ & $2.9777(-3)$ & $2.4963(-3)$ & $1.7072(-4)$ \\
1.6 & & $1.2498(-2)$ & $2.8919(-3)$ & $2.2171(-3)$ & $1.6963(-4)$ \\
1.7 & & $1.1158(-2)$ & $2.7898(-3)$ & $1.9516(-3)$ & $1.6819(-4)$ \\
1.8 & & $9.8391(-3)$ & $2.6849(-3)$ & $1.7123(-3)$ & $1.6678(-4)$ \\
1.9 & & $8.6095(-3)$ & $2.5735(-3)$ & $1.4960(-3)$ & $1.6521(-4)$ \\
2.0 & & $7.4791(-3)$ & $2.4546(-3)$ & $1.3015(-3)$ & $1.6371(-4)$ \\
\hline
\end{tabular}

\section{Positron-Hydrogen Scattering}

It should be noted that for positron-hydrogen scattering $\Phi^{p o l}$ has a positive sign instead of the negative sign as in the case of electron-hydrogen scattering:

$$
\Phi^{p o l}\left(\vec{r}_{1}, \vec{r}_{2}\right)=\phi_{0}\left(\vec{r}_{2}\right)+\frac{\chi_{S T}\left(r_{1}\right)}{r_{1}^{2}} \frac{u_{1 s \rightarrow p}\left(r_{2}\right)}{r_{2}} \frac{\cos \left(\theta_{12}\right)}{\sqrt{\pi Z}} \text {. }
$$

In Table 13, we compare phase shift for $S$-wave scattering obtained in [42] using 35-term correlation functions with those obtained using 84-term correlation functions where projection operators were used [43]. With shorter expansions, improved results have been obtained, as also indicated in Figure 3 from Ref. [42].

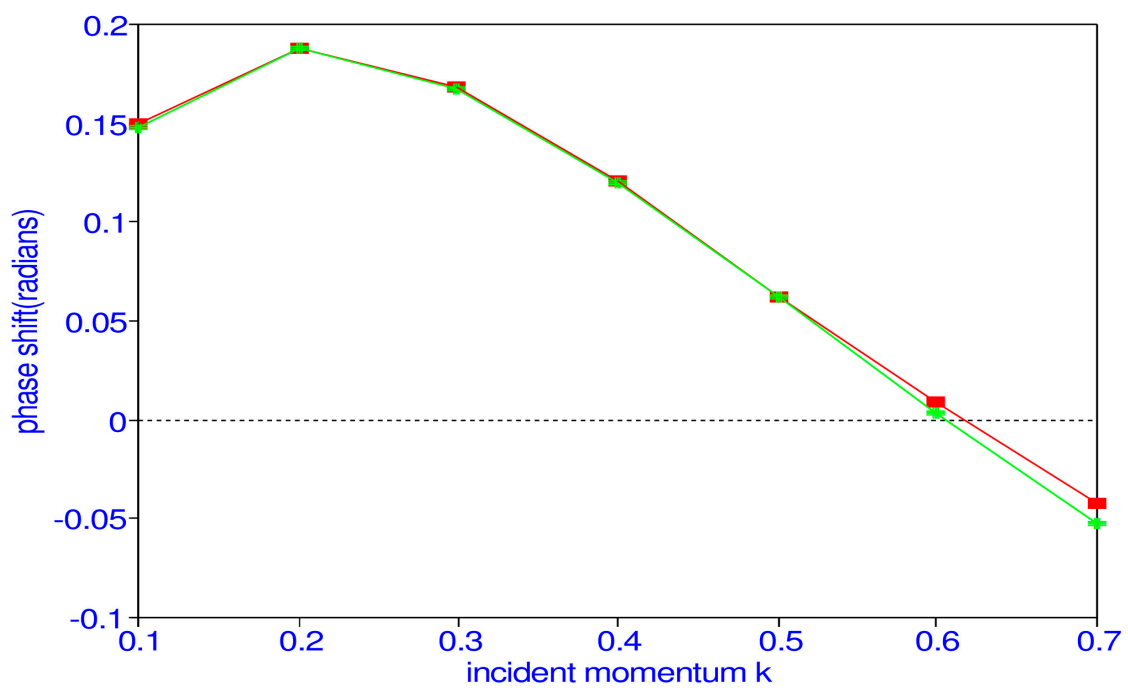

Figure 3. (Color online) The upper curve represents the present results, while the lower curve represents obtained by Bhatia et al. [43]. 
The scattering length is -2.10258 . Using the correction given in Equation (54), we find the scattering length is -2.104004 which agrees with $-21036 \pm 0.0004$ obtained by Houston and Drachman [44], and with $-2.103 \pm 0.001$ obtained by Humberston and Wallace [45].

Table 13. Comparison of phase shifts (radians) for $\mathrm{e}^{+}-\mathrm{H}$ scattering for various $k$ obtained in [42] with those obtained in [43].

\begin{tabular}{cccc}
\hline$k$ & Hybrid Theory [42] & P, Q Projections [43] & $Z_{\text {eff }}$ \\
\hline 0.1 & 0.14918 & 0.1483 & 8.092 \\
0.2 & 0.18803 & 0.1877 & 5.357 \\
0.3 & 0.16831 & 0.1677 & 4.264 \\
0.4 & 0.12083 & 0.1201 & 3.370 \\
0.5 & 0.06278 & 0.0624 & 2.424 \\
0.6 & 0.00903 & 0.0039 & 2.249 \\
0.7 & -0.04253 & -0.0512 & 2.069 \\
\hline
\end{tabular}

10. $Z_{\text {eff }}$

There is a possibility of positronium annihilation for which the cross section is given by:

$$
\sigma_{a}\left(\pi a_{0}^{2}\right)=Z_{e f f} \alpha^{3} / k
$$

Annihilation takes place with the emission of two gamma rays [46]. For hydrogen:

$$
Z_{e f f}=\iint d \vec{r}_{1} d \vec{r}_{2}\left|\Psi_{L}\left(\vec{r}_{1}, \vec{r}_{2}\right)\right|_{2} \delta\left(\vec{r}_{1}, \vec{r}_{2}\right)
$$

The normalization of the scattering function for $r_{1} \rightarrow \infty$ is given by:

$$
u_{L}\left(r_{1}\right)=[4 \pi(2 L+1)]^{0.5} \sin \left(k r_{1}-\frac{L \pi}{2}+\eta\right) / k
$$

\section{Positronium Formation}

Positronium formation takes place when the incident positron captures an electron of the hydrogen target:

$$
e^{-}+H(1 s) \rightarrow P s+P
$$

$Z_{\text {eff }}$ and positronium formation have been calculated [42] using hybrid theory. The results for $Z_{\text {eff }}$ are given in Table 13 and positronium formation cross sections are given in [42]. A similar calculations have been carried out for $P$-wave scattering. $Z_{\text {eff }}$ and positronium formation cross sections have also been calculated [47].

\section{Conclusions}

In summary, we have used an approximation for scattering calculations in which the long-range and short-range correlations have been included at the same time variationally. The phase shifts for electron and positron scattering have a lower bound to the exact phase shifts. We have used these continuum functions to calculate resonance parameters, photoabsorption, and excitation cross sections, $Z_{\text {eff }}$ and positronium formation cross sections. The results are in agreement with those obtained in other calculations.

Acknowledgments: Thanks are extended to R.J. Drachman for discussions on low-energy scattering.

Conflicts of Interest: The author declares no conflict of interest. 


\section{References}

1. Morse, P.M.; Allis, W.P. The effect of Exchange on the Scattering of Slow Electrons from Atoms. Phys. Rev. 1933, 44, 269. [CrossRef]

2. Kohn, W. Variational Methods in Nuclear Collision Problems. Phys. Rev. 1948, 74, 1763. [CrossRef]

3. Schwartz, C. Electron Scattering from Hydrogen. Phys. Rev. 1961, 124, 1468. [CrossRef]

4. Burke, P.G.; Smith, K. The Low-Energy Scattering of Electrons and Positrons by Hydrogen Atoms. Rev. Mod. Phys. 1962, 34, 458. [CrossRef]

5. Burke, P.G.; Robb, W.D. The R-matrix Theory of Atomic Processes. Adv. At. Mol. Phys. 1976, 11, 143.

6. Temkin, A.; Lamkin, J.C. Application of the Method of Polarized Orbitals to the scattering of Electrons from Hydrogen. Phys. Rev. 1961, 121, 788. [CrossRef]

7. Bhatia, A.K.; Temkin, A. Complex-correlation Kohn T-matrix method of calculating total and elastic cross sections: Electron-hydrogen elastic scattering. Phys. Rev. A 2001, 64, 032709. [CrossRef]

8. Wigner, E.P. On the Behavior of Cross Sections Near Threshold. Phys. Rev. 1948, 73, 1002. [CrossRef]

9. Temkin, A. A note on the Scattering of Electrons from Atomic Hydrogen. Phys. Rev. 1959, 116, 358. [CrossRef]

10. Sloan, I.H. The method of polarized orbitals for the elastic scattering of slow electrons by ionized helium and atomic hydrogen. Proc. R. Soc. Lond. A 1964, 281, 151-163. [CrossRef]

11. Feshbach, H. A unified theory of nuclear reactions. II. Ann. Phys. 1962, 19, 287-313. [CrossRef]

12. Bhatia, A.K.; Temkin, A. Symmetric Euler-Angle Decomposition of the Two-Electron Fixe-Nucleus Problem. Rev. Mod. Phys. 1964, 36, 1050. [CrossRef]

13. Bhatia, A.K. Hybrid theory of electron-hydrogen elastic scattering. Phys. Rev. A 2007, 75, 032713. [CrossRef]

14. Shertzer, J.; Temkin, A. Direct calculation of the scattering amplitude without partial-wave analysis III. Inclusion of correlation effects. Phys. Rev. A 2006, 74, 052701. [CrossRef]

15. Scholz, T.; Scott, P.; Burke, P.G. Electron-hydrogen-atom scattering at intermediate energies. J. Phys. B 1988, 21, L139. [CrossRef]

16. Bhatia, A.K. Applications of the hybrid theory in the scattering of electrons from $\mathrm{He}^{+}$and $\mathrm{Li}^{2+}$ and resonances in these systems. Phys. Rev. A 2008, 77, 052707. [CrossRef]

17. Gien, T.T. Accurate calculation of phase shifts for electron-He ${ }^{+}$collisions. J. Phys. B 2002, 35, 4475. [CrossRef]

18. Gien, T.T. Accurate calculation of phase shifts for electron collisions with positive ions. J. Phys. B 2003, 36, 2291. [CrossRef]

19. Bhatia, A.K. Hybrid theory of P-wave electron-hydrogen elastic scattering. Phys. Rev. A 2012, 85, 052708. [CrossRef]

20. Bhatia, A.K. Application of $P$-wave hybrid theory in the scattering of electrons from $\mathrm{He}^{+}$and resonances in He and $\mathrm{H}^{-}$. Phys. Rev. A 2012, 86, 032709. [CrossRef]

21. Botero, J.; Shertzer, J. Direct numerical solution of the Schrödinger equation for the quantum scattering problems. Phys. Rev. A 1992, 46, R1155. [CrossRef] [PubMed]

22. Bhatia, A.K. D-wave electron-H, $\mathrm{He}^{+}$, and $\mathrm{Li}^{2+}$ elastic scattering and photoabsorption in $P$ states of two-electron system. Phys. Rev. A 2014, 89, 062720. [CrossRef]

23. Temkin, A. Polarization and Triplet Electron-Hydrogen Scattering Length. Phys. Rev. Lett. 1961, 6, 354. [CrossRef]

24. Bhatia, A.K.; Temkin, A. Calculation of autoionization of $\mathrm{He}$ and $\mathrm{H}^{-}$using the projection-operator formalism. Phys. Rev. A 1975, 11, 2018. [CrossRef]

25. O'Malley, T.F.; Rosenberg, L.; Spruch, L. Low-Energy Scattering of a charged Particle by a Neutral Polarizable System. Phys. Rev. 1962, 125, 1300. [CrossRef]

26. Wildt, R. Electron Affinity in Astrophysics. Astrophys. J. 1939, 89, 295-301. [CrossRef]

27. Bhatia, A.K. Hybrid theory of $P$-wave electron- $\mathrm{Li}^{2+}$ elastic scattering and photoabsorption in two-electron systems. Phys. Rev. A 2013, 87, 042705. [CrossRef]

28. Bell, K.L.; Kingston, A.E. The bound-free absorption coefficient of the negative hydrogen ion. Proc. Phys. Soc. Lond. 1967, 90, 895. [CrossRef]

29. Ohmura, T.; Ohmura, H. Electron-Hydrogen Scattering at low energies. Phys. Rev. 1960, 118, 154. [CrossRef]

30. Nahar, S.N. New Quests in Stellar Astrophysics. II. The Ultraviolet Properties of Evolved Stellar Populations; Chavez, M., Bertone, E., Rosa-Gonzalez, D., Rodriguez-Merino, L.H., Eds.; Springer: New York, NY, USA, 2009 ; p. 245. 
31. Samson, J.A.R.; He, Z.X.; Yin, L.; Haddad, G.H. Precision measurements of the absolute photoionization cross sections of He. J. Phys. B 1994, 27, 887. [CrossRef]

32. Burke, P.G.; Schey, H.M.; Smith, K. Collisions of Slow Electrons and Positrons with Atomic Hydrogen. Phys. Rev. 1963, 129, 1258. [CrossRef]

33. Callaway, J. Scattering of electrons by atomic hydrogen at intermediate energies: Elastic Scattering and $n=2$ excitation from 12 to $54 \mathrm{eV}$. Phys. Rev. A 1985, 32, 775. [CrossRef]

34. Callaway, J.; Unnikrishnan, K.; Oza, D.H. Optical potential of electron-hydrogen scattering at intermediate energies. Phys. Rev. A 1987, 36, 2576. [CrossRef]

35. Scott, M.P.; Scholz, T.T.; Walters, H.R.; Burke, P.G. Electron scattering by atomic hydrogen at intermediate energies: Integrated elastic, is-2s and 1s-2p cross sections. J. Phys. B 1989, 22, 3055. [CrossRef]

36. Poet, R. The exact solution for a simplified model of electrons scattering by hydrogen atoms. J. Phys. B 1978, 11, 3081. [CrossRef]

37. Lloyd, M.D.; McDowell, M.R.C. An application of the polarized approximation to electron-impact excitation of atomic hydrogen. J. Phys. B 1969, 2, 1313. [CrossRef]

38. Bhatia, A.K. Excitation of the $2 S$ State of Atomic Hydrogen by Electron Impact. Atoms 2018, 6, 7. [CrossRef]

39. Kauppila, W.E.; Ott, W.R.; Fite, W.L. Excitation of Atomic Hydrogen to the Metastable $2^{2} S_{1 / 2}$ State by Electron-Impact. Phys. Rev. A 1970, 1, 1099. [CrossRef]

40. Bhatia, A.K.; Drachman, R.J. Photoejection with excitation in $\mathrm{H}^{-}$and other systems. Phys. Rev. A 2015, 91, 012702. [CrossRef]

41. Lallement, R.; Que'merais, E.; Bertaux, J.-L.; Sandel, B.R.; Izmodenov, V. Voyager Measurements of Hydrogen Lyman- $\alpha$ Diffuse Emission from the Milky Way. Science 2011, 334, 1665-1669. [CrossRef] [PubMed]

42. Bhatia, A.K. Positron-Hydrogen Scattering, Annihilation, Positronium Formation. Atoms 2016, 4, 27. [CrossRef]

43. Bhatia, A.K.; Temkin, A.; Drachman, R.J.; Eiserike, H. Generalized Hylleraas Calculation of PositronHydrogen Scattering. Phys. Rev. A 1971, 3, 1328. [CrossRef]

44. Houston, S.K.; Drachman, R.J. Positron-Atom by Kohn and Harris Methods. Phys. Rev. A 1971, $3,1335$. [CrossRef]

45. Humberston, J.W.; Wallace, J.B.G. The elastic scattering of positrons by atomic hydrogen. J. Phys. B 1972, 5, 1138. [CrossRef]

46. Ferrall, R.A. Theory of Positron Annihilation in Solids. Rev. Mod. Phys. 1956, 28, 308. [CrossRef]

47. Bhatia, A.K. P-wave Positron-Hydrogen Scattering, Annihilation, and Positronium Formation. Atoms 2017, 5, 17. [CrossRef]

(C) 2018 by the author. Licensee MDPI, Basel, Switzerland. This article is an open access article distributed under the terms and conditions of the Creative Commons Attribution (CC BY) license (http://creativecommons.org/licenses/by/4.0/). 